\section{Procesos de integración en salud: una reflexión sobre los desafíos del MERCOSUR en salud}

\author{
Health integration processes: challenges for \\ MERCOSUR in the health field
}

\author{
${ }^{1}$ Grupo de Estudios en \\ Economía, Organización \\ y Políticas Sociales, \\ Montevideo, Uruguay. \\ Correspondencia \\ D. M. Sánchez \\ Grupo de Estudios en \\ Economía, Organización y \\ Políticas Sociales. \\ Francisco Vidal 643, apto. 102, \\ Montevideo 11300, Uruguay. \\ dibarsan@adinet.com.uy
}

\begin{abstract}
This paper describes the institutional background in Latin American integration in both the economy and health, and proposes a systematization of possible health integration modalities. Facilitating and inhibiting factors for integration according to each modality are identified, and their feasibility is discussed in the present context. The structure and functioning of MERCOSUR health structures (Ministerial Meeting and Sub-group 11) are briefly described, as well as the advances achieved to date, reflecting on the possible causes of uneven progress in different areas.
\end{abstract}

Health Systems; Health Services; Intersectorial Action

\section{Antecedentes}

El presente trabajo, presentado en el Foro MERCOSUR sobre Integración Regional y Servicios de Salud, organizado por la Red de Investigación en Sistemas y Servicios de Salud del Cono Sur en marzo de 2006, recoge la experiencia de la autora como integrante de la delegación uruguaya en la Subcomisión de Evaluación de Tecnologías Sanitarias y la Comisión de Servicios de Salud del Subgrupo de Trabajo 11 Salud (SGT 11) del MERCOSUR, durante varios años y las conclusiones que surgen de esa práctica. Pretende ser una instancia de reflexión sobre la misma, a la luz de las dificultades que el proceso de integración regional presenta en el momento actual. No se realiza un ejercicio teórico sobre "la integración", objeto abstracto, sino sobre una experiencia concreta que ha tenido avances y retrocesos desde su comienzo más bien reciente.

La historia de la integración, o al menos su búsqueda, es antigua en América Latina. Pueden encontrarse referencias a ella desde las ideas federales y pan-americanistas de varios de los héroes de la Independencia en el siglo XIX, que se vieron frustradas en la constitución de Estados a veces enfrentados entre sí, hasta nuestros días.

Ya en el siglo XX se crearon varias estructuras orientadas a la integración política y económica, siendo las más relevantes las que se citan a continuación: 
a) La Organización de Estados Americanos (OEA), creada en 1948 con una vocación de integración política y que cuenta actualmente con 35 países miembros (http://www.oea.org, accedido el 20/Mar/2006).

b) La Asociación Latinoamericana de Libre Comercio (ALALC), creada en 1960 con el objetivo de constituir una zona de libre comercio en un plazo de 12 años. Integrada por 11 países, fue la arena de discusión de dos visiones opuestas: una de "integración acelerada" propiciada por Chile, Venezuela y Colombia; y una de "integración lenta”, propiciada por Argentina, Brasil y México y finalmente adoptada. Fue la antecesora de la Asociación Latinoamericana de Integración (ALADI - http://www.aladi.org, accedido el 20/ Mar/2006).

c) La ALADI, integrada por 12 países, fue creada en 1980 y cuenta como instrumentos de integración con los acuerdos de alcance parcial, los acuerdos de complementación económica y los acuerdos regionales, entre los últimos la preferencia arancelaria regional.

d) La Comunidad Andina de Naciones (CAN), creada en 1996, pero sucesora del Grupo Andino, constituido en 1969. En sus orígenes el Grupo Andino tuvo un objetivo de integración en el desarrollo, propiciando una política de sustitución de importaciones, lo que se modificó formalmente en 1987. En 1993 se constituyó en una zona de libre comercio con un arancel externo común a partir de 1993. La CAN ha visto cambios en su integración, siendo el último de ellos el anuncio por parte de Venezuela de abandonar este bloque (http:/ / www.can.org, accedido el 20/Mar/2006). e) En lo atinente a integración en salud, la Organización Panamericana de la Salud, creada en 1902 con el nombre de Oficina Sanitaria Panamericana, se convirtió luego en la representante para la región de las Américas de la Organización Mundial de la Salud, pero manteniendo su marcado carácter panamericanista. A su vez, la Región Andina cuenta con la estructura del Convenio Hipólito Unanue desde 1971.

A pesar de que las estructuras previas no fueron exitosas en avanzar el proceso de integración del continente, en el imaginario colectivo del Cono Sur, el MERCOSUR sí tendría la capacidad de hacerlo. Sin embargo, el bloque ha sido marco de graves conflictos desde su comienzo y, al menos desde la visión de los socios más pequeños, existe hoy un fuerte cuestionamiento de su utilidad y su capacidad de resolver potenciales conflictos. A pesar de la relevancia que se le ha asignado en materia de posicionamiento internacional para los países que lo integran, el proceso de creación y consolidación del MERCOSUR no ha sido objeto de tanta investigación como podría esperarse, al menos desde el punto de vista de su impacto sobre la salud y los sistemas de salud. Se recurre quizás con demasiada frecuencia al paralelismo con la constitución de la Comunidad Europea, implicando de alguna forma que el europeo es un camino inevitable y no solamente una de entre muchas formas de organización posibles.

En una revisión de las bases de datos SciELO y LILACS con las palabras claves integración y MERCOSUR, sin límite de tiempo, se encontró un número muy limitado de artículos que analizaban la experiencia del MERCOSUR desde los puntos de vista de los recursos humanos 1 , el mercado de medicamentos 2, y la integración de servicios de salud 3 . El resto del material hallado se centra en las posibilidades de integración regional en un contexto de globalización y la relación con la potencia hegemónica a nivel mundial, pero sin profundizar en las grandes diferencias de posibilidades e intereses intra-regionales. Un interesante trabajo de Hirst 4 identificó a los distintos actores del proceso de integración, así como los universos temáticos que generan controversias entre y dentro de los países de la sub-región (economía política internacional, economía política doméstica, política exterior y de seguridad internacional y opciones de institucionalización del proceso asociativo) 4 .

No deja de ser sintomático que la mayor parte de la bibliografía hallada tenga aproximadamente diez años, correspondiendo al período de grandes expectativas respecto al bloque y no al momento actual en que ya es posible evaluar algunos de sus efectos.

\section{Cronología y estructura del MERCOSUR salud}

El Tratado de Asunción, del 26 de marzo de 1991, creó formalmente el MERCOSUR. Según el texto de dicho Tratado, el propósito de este acuerdo es la constitución de un Mercado Común, que debía estar conformado el 31 de diciembre de 1994. En el artículo 1 del Tratado, el Mercado Común implica:

a) La libre circulación de bienes, servicios y factores productivos entre los países.

b) El establecimiento de un arancel externo común y la adopción de una política comercial común.

c) La coordinación de políticas macroeconómicas y sectoriales de comercio exterior, agrícola, industrial, fiscal, monetaria, cambiaria y de capitales, de servicios, aduanera, de transportes y comunicaciones y otras que se acuerden.

d) El compromiso de los Estados partícipes de armonizar sus legislaciones en las áreas perti- 
nentes para lograr el fortalecimiento del proceso de integración.

Los órganos creados por el tratado fueron dos: el Consejo del Mercado Común (CMC), encargado de la conducción política e integrado por los ministros de relaciones exteriores y de economía de los estados partícipes, y el Grupo Mercado Común (GMC), órgano ejecutivo coordinado por los ministerios de relaciones exteriores e integrado por estos ministerios, así como por los ministerios de economía o sus equivalentes y los bancos centrales de los cuatro Estados partícipes.

El tema salud se incluyó en la discusión, dentro del SGT 3 Normas Técnicas, en una comisión llamada Productos de Salud por la Resolución GMC no. 51 del año 1992.

El 16 de diciembre de 1994 se firmó el Tratado de Ouro Preto, que amplió el número de órganos del MERCOSUR a seis: (1) el CMC; (2) el GMC; (3) la Comisión de Comercio del MERCOSUR (CCM); (4) la Comisión Parlamentaria Conjunta (CPC); (5) el Foro Consultivo Económico-Social (FCES); (6) la Secretaría Administrativa del MERCOSUR (SAM).

En el año 1995 se creó la Reunión de Ministros de Salud, a través de la Decisión del CMC $n^{\circ}$. 03/1995.

La 1a Reunión de Ministros de Salud del MERCOSUR, realizada el 7 de noviembre de 1995, solicitó al GMC que se invite a participar - en carácter de observadores- a los Ministros de Salud de Bolivia y Chile, y a la Organización Panamericana de Salud como organismo técnico - asesor.

En 1996 el GMC aprobó la creación del SGT 11, a través de la Resolución GMC no. 151/1996 y estableció y aprobó su primera pauta de trabajo a través de la Resolución GMC no . 04/1998.

En este momento se encuentra vigente una pauta negociadora de diciembre de 2004, que identifica como tarea general: armonizar legislaciones y coordinar acciones entre los Estados partícipes referentes a la atención a la salud, bienes, servicios, materias primas y productos del área de salud, vigilancia epidemiológica, vigilancia en salud y control sanitario, con la finalidad de promover y proteger la salud, la vida de las personas y eliminar los obstáculos al comercio regional, a la atención integral y de calidad, contribuyendo de esta manera al proceso de integración.

Tanto la Reunión de Ministros como el SGT 11 tienen a su vez una compleja estructura interna, como se aprecia en las Figuras 1 y 2.

El estado de avance del trabajo en todas estas áreas ha sido desigual; mientras la comisión de vigilancia en salud ha continuado procesos de larga data, simplemente con nuevos instrumen- tos institucionales, la comisión de productos de salud ha avanzado especialmente en la temática de cosméticos, y en la producción de normativa sobre buenas prácticas de fabricación de medicamentos y la formación de inspectores para su verificación. La Comisión de Servicios de Salud, que es en la que se centra este trabajo, ha tenido mayor dificultad en determinar sus competencias y avanzar, lo que refleja la dificultad de la integración en el tema, que parte ya de su propia conceptualización.

La participación de los países que tienen carácter de observadores también ha sido despareja, si bien participan de forma consistente en las reuniones de ministros, Bolivia no lo ha hecho en el SGT 11 y Chile solamente lo ha hecho en temas puntuales de su interés particular, especialmente en lo concerniente a vigilancia de fronteras. Venezuela ha solicitado su integración en el MERCOSUR. Por ese motivo los comentarios de este artículo corresponden a la actuación de los miembros plenos del bloque.

\section{Objetivos posibles en la integración de servicios de salud}

A diferencia del comercio de bienes, el modelo de integración de servicios de salud no ha sido definido previamente, por lo que la comisión ha avanzado en distintos momentos de su actuación con supuestos diferentes.

Los modelos posibles de integración en salud pueden concebirse de distintas formas, según se presenta a continuación:

a) Coordinación de servicios en zonas fronterizas. Este es el modelo menos ambicioso, ya que se restringe en lo geográfico, no pretendiendo alterar el funcionamiento de las estructuras en el resto del país.

b) Integración de servicios en zonas fronterizas. La integración supone varios pasos más allá de la simple coordinación, ya que subyace la idea de una dirección conjunta.

c) Libre tránsito de personas: profesionales de la salud. El libre tránsito de profesionales implica no solamente el reconocimiento por las autoridades de los países de los títulos de grado expedidos en los demás, sino también de los de postgrado. La dificultad aquí radica en que no existen las mismas especialidades en los cuatro países, y además los mecanismos para obtener los títulos de post-grado también son diferentes. Por otra parte, la habilitación para el ejercicio por la autoridad nacional, si bien es suficiente en Uruguay y Paraguay, no lo es en Argentina y Brasil, países federativos y con modelo de colegiación de las profesiones. 


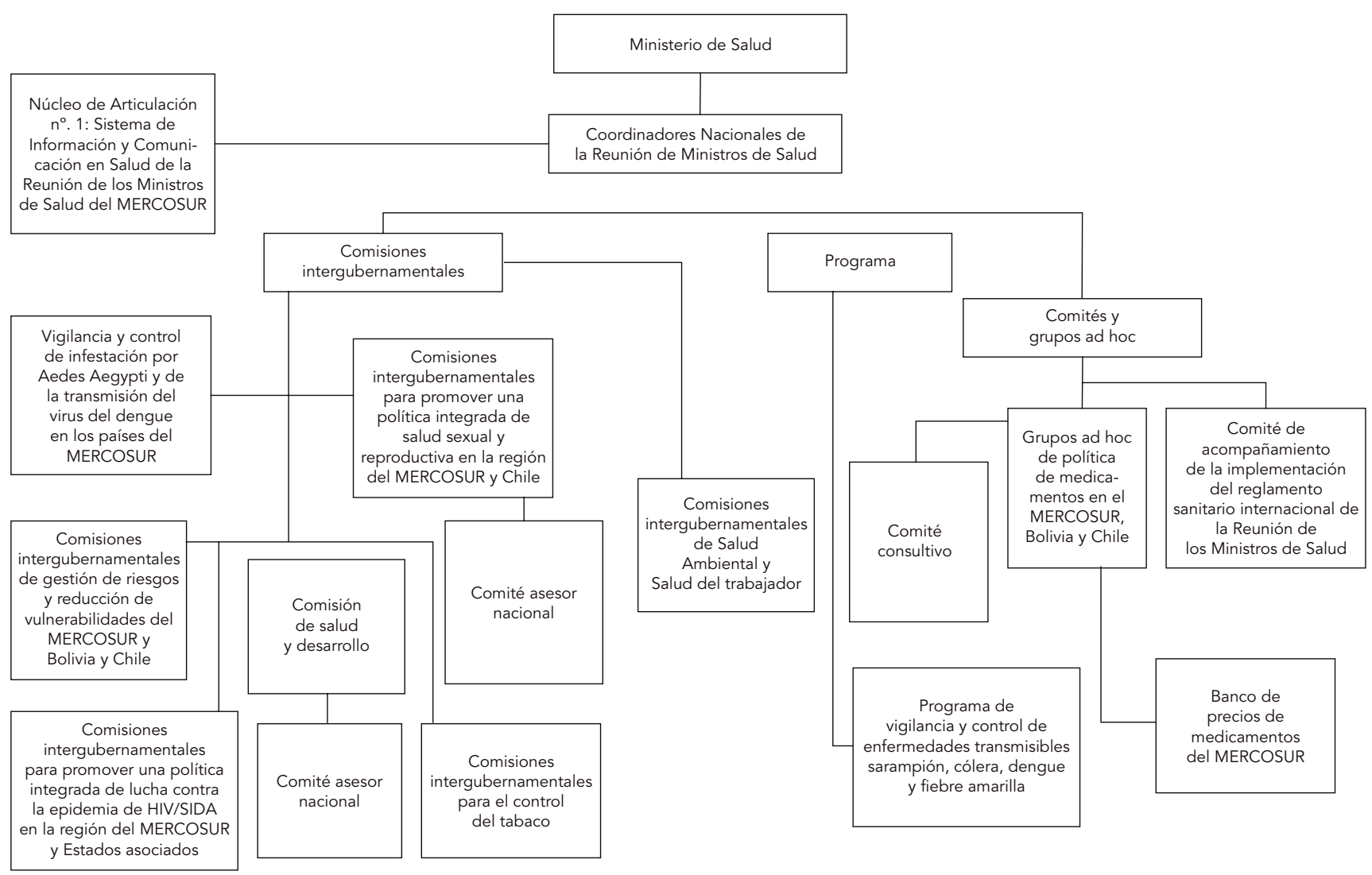

Fuente: Sistema de Información en Salud del MERCOSUR (http://www.mercosulsalud.org/espanhol/reuniones/index.htm).

d) Libre tránsito de personas: los usuarios del sistema de salud. La forma más sencilla de migración es la del turista o residente no permanente. En este momento los derechos de los ciudadanos del MERCOSUR en materia de salud solamente pueden ser ejercidos dentro de su propio territorio, no existiendo portabilidad de derechos. No se ha discutido aún siquiera la posibilidad de ejercer el derecho a la atención en todo el MERCOSUR a nivel de urgencias y emergencias.

En el momento en que exista libre tránsito de personas y los trabajadores puedan migrar indistintamente de un país a otro para trabajar, se planteará el problema de quién es responsable de su atención de salud y quién debe hacerse cargo de sus cotizaciones. A esto le sigue qué sucederá con los derechos adquiridos en el país donde trabajó y cotizó, si ese trabajador regresa a su país o a otro del bloque. Este tema se engloba con el de la seguridad social en su conjunto. Si las prestaciones cubiertas por los sistemas de salud de los dos países fueran diferentes, ¿̇a cuáles tendrá acceso ese ciudadano?

e) Libre tránsito de servicios. Existen también aquí dos casos posibles: (i) un servicio habilitado en el país A que desee instalarse también en el país B. En esta situación es bastante claro que cada país aplicará su propia legislación para la habilitación de servicios, que en la práctica funcionarán como entidades independientes sujetas a las normas locales; (ii) compra de servicios de salud en el país A por el sistema de salud del país B, para suplir un déficit de algún tipo. Se debe decidir en esta situación las normas a las se debe ajustar el servicio brindado. 


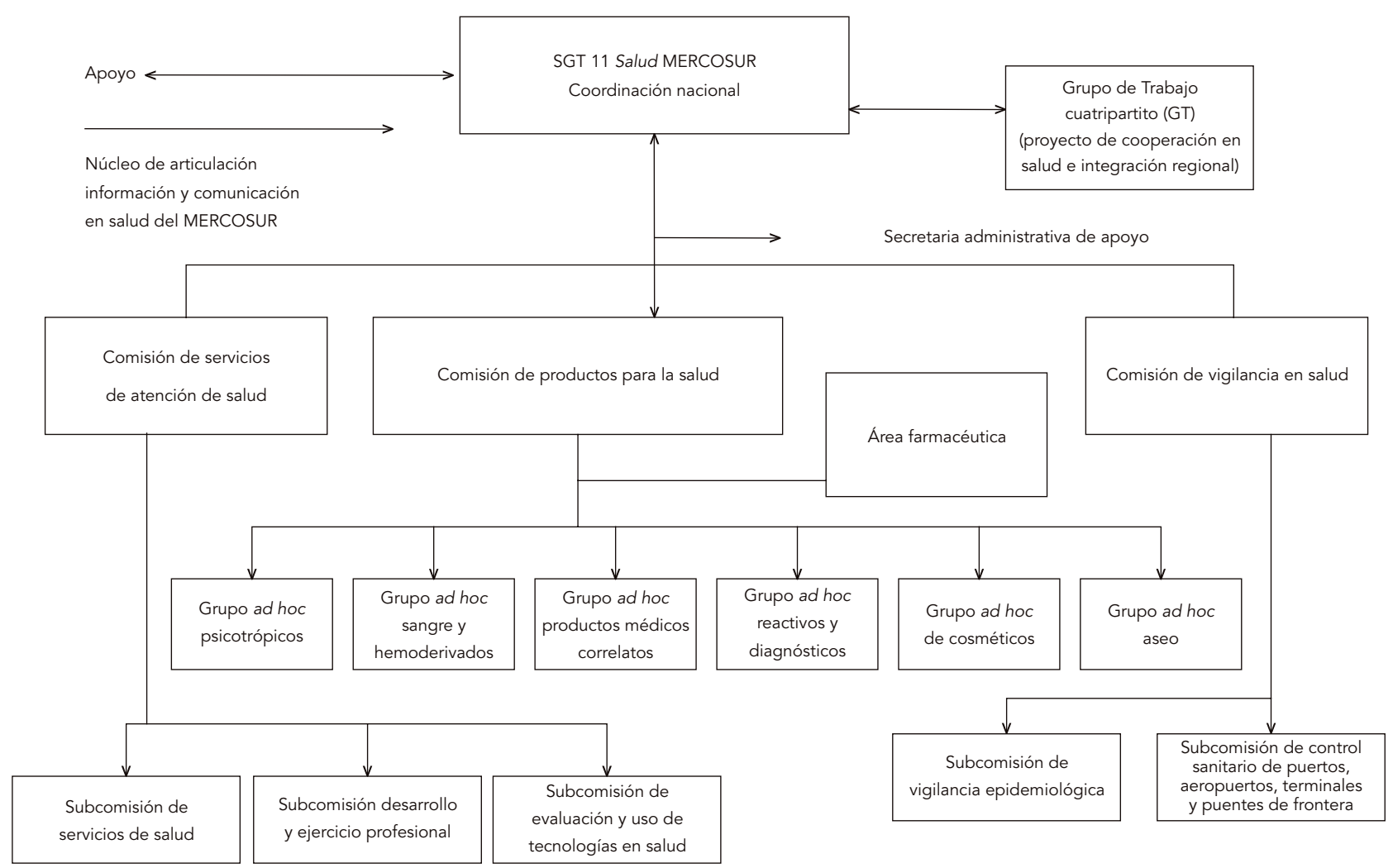

Fuente: Sistema de Información en Salud del MERCOSUR (http://www.mercosulsalud.org/espanhol/sgt/Plano_Anual/ORG_SGT.ppt).

\section{Dificultades estructurales para la integración}

En estos momentos nos encontramos aún en una etapa de estudio de la coordinación de servicios en zonas fronterizas. Si bien los modelos de integración señalados antes no conforman una sucesión obligatoria, es de suponer que de avanzar el proceso de integración económica, será necesario avanzar en la discusión de la prestación de servicios sociales, entre ellos la salud, a imagen de la experiencia europea 5 . Las autoridades de los Estados partícipes conocen esto bien, y sin embargo, hasta donde sabemos, no se encuentran en discusión respuestas a estos desafíos de un futuro no tan lejano; corresponde preguntarse por qué. Una respuesta posible es que se apuesta a que la fuerza de los hechos determine la integración a nivel de servicios, antes que discutir abiertamente sus principales obstáculos por ser puntos de gran sensibilidad.
Se mencionan a continuación algunos de estos problemas:

- El contexto político del proceso de integración requiere como condición sine qua non un nivel de estabilidad política importante, y el mantenimiento de reglas de juego claras a nivel de política internacional y de política económica, independientemente de los gobiernos del momento. A pesar de ser uno de los pilares de la integración económica, no se aplica el Arancel Externo Común a partir de la crisis que sufriera Argentina en 2001 y por otra parte, una parte de la frontera entre Argentina y Uruguay se encontró bloqueada por ciudadanos argentinos desde diciembre de 2005 hasta el mes de marzo de 2006 y nuevamente en la semana del 9 al 16 de abril de 2006, debido a un conflicto derivado de la instalación de dos plantas de celulosa en la margen uruguaya del río Uruguay. Esta situación ha deteriorado la relación entre los dos países y en estas condiciones el discurso de integración se vuelve por lo 
menos dudoso. A ello se suma la doble pertenencia de los países a varios bloques de naciones y a estructuras supra-regionales como ALADI o la OEA, y la firma de Tratados de Libre Comercio con países fuera de la región, como México.

- Estructura demográfica y condición socioeconómica; en la Tabla 1 se presentan los principales indicadores demográficos y económicos de los Estados partícipes del MERCOSUR, así como los de los observadores y Venezuela, que en el mes de marzo de 2006 se encontraba en proceso de integración al bloque. Como puede apreciarse, existen diferencias sustanciales tanto en el tamaño de las poblaciones como en el de sus economías, lo que se refleja en la relación de poder entre países y en la capacidad de adaptarse a los cambios normativos que el MERCOSUR propone. Los indicadores de equidad, tal como la relación entre los ingresos del $20 \%$ más rico y el $20 \%$ más pobre, o los porcentajes de población por debajo de la línea de pobreza, también muestran marcadas diferencias intra-regionales, que no se corresponden necesariamente con los niveles de riqueza global de los países considerados. Condiciones tan diversas hacen difícil concebir respuestas comunes, si se pretende ir más allá de los enunciados genéricos.

- Condiciones de salud y estructura de los sistemas de salud. La Tabla 2 nos muestra un resumen de algunos de los principales indicadores de salud en los países del MERCOSUR. Al presentarse datos globales se ocultan las diferencias regionales y por grupos de población especialmente vulnerables. Igualmente, se comprueba una marcada diferencia en los indicadores de salud de los países del bloque, tanto en lo que respecta a tasas de mortalidad infantil y materna como en las tasas de mortalidad por enfermedades transmisibles y crónico-degenerativas. Estas diferencias surgen de una historia de políticas públicas distintas y a su vez determinan formas de organización de los servicios diferentes para dar respuesta a realidades diversas.

En cuanto a la estructura de los sistemas de salud, mientras Brasil cuenta con un Sistema Único de Salud (aunque con efectores públicos y privados) y la misma Constitución garantiza el acceso de todos los ciudadanos a todos los servicios que pudieran necesitar, los restantes Estados partícipes cuentan con sistemas mixtos público-privado, y con diferencias en las prestaciones cubiertas, según la institución a la que se pertenezca.

La Tabla 3 muestra algunos indicadores de recursos destinados a la salud en los países de la región, los que también presentan marcadas diferencias, con el consiguiente impacto sobre la posibilidad de armonizar criterios relativos a la organización de los sistemas y servicios de salud.

- Estructuras estatales federales y centralistas. Mientras Argentina y Brasil son estados federales, con multiplicidad de actores y niveles de decisión, Paraguay y Uruguay son países con gobiernos centrales fuertes, en los que radican todas las competencias necesarias para resolver sobre los temas de salud. Los Estados federales no solamente presentan una multiplicidad de actores gubernamentales, sino también en la estructura de la sociedad civil, particularmente las estructuras gremiales de los profesionales de la salud, que repiten la organización estatal/provincial y nacional, existiendo múltiples interlocutores, mientras que en los países centralizados las estructuras gremiales también se encuentran centralizadas.

\section{Consecuencias}

La falta de una definición previa del modelo de integración en salud que se desea a largo plazo, así como la diversidad entre países y la falta de avance en la integración económica, han tenido importantes efectos en el desarrollo del proceso de integración en salud en el MERCOSUR.

Lo primero a destacar es el gran número de temas que han quedado a discusión en el ámbito de la reunión de ministros. Esta es una aceptación de la dimensión política de los mismos, pero también ha significado que en muchos de ellos no se haya podido avanzar más allá del nivel de encargar estudios sobre los distintos temas. Por su parte, el SGT 11 que se plantea como una estructura con perfil más técnico, aunque siguiendo directivas políticas, ha recurrido en numerosas ocasiones a la elevación para discusión a nivel de reunión de ministros cuando no era posible obtener consenso en un tema. Probablemente esto explique la aparente duplicación de grupos temáticos en ambos niveles (Reunión de Ministros y SGT 11).

Lo segundo es el desarrollo mayor de la discusión en aquellos temas directamente vinculados al comercio de bienes (productos de salud), ante aquellos cuyo objetivo es el aseguramiento de derechos de los ciudadanos (servicios de salud).

En lo relativo al trabajo de la Comisión de Servicios de Atención a la Salud, la diversidad y la falta de una imagen objetiva consensuada del modelo de integración que se pretende alcanzar han llevado a las siguientes situaciones:

a) Sub Comisión de Servicios de Salud. Su principal actividad es la formulación de normas comunes de habilitación de servicios de salud. Ha pasado por distintas etapas en las que privile- 
Principales indicadores demográficos y socio-económicos en los países del MERCOSUR, información en el último año disponible.

\begin{tabular}{|c|c|c|c|c|c|c|c|}
\hline \multirow[t]{2}{*}{ Indicador } & \multicolumn{4}{|c|}{ Estados partícipes } & \multicolumn{2}{|c|}{ Observadores } & \multirow{2}{*}{$\begin{array}{c}\text { En proceso } \\
\text { de } \\
\text { integración } \\
\text { Venezuela }\end{array}$} \\
\hline & Argentina & Brasil & Paraguay & Uruguay & Bolivia & Chile & \\
\hline Población total (miles, 2005) & 38.747 & 186.405 & 6.158 & 3.463 & 9.182 & 16.295 & 26.749 \\
\hline \% población urbana & 90,6 & 84,2 & 58,3 & 93,0 & 64,4 & 87,7 & 88,1 \\
\hline Esperanza de vida al nacer (total, 2005) & 74,9 & 71,3 & 71,5 & 75,9 & 64,9 & 78,3 & 73,4 \\
\hline \% población alfabetizada (2005) & 97,2 & 88,9 & 94,4 & 98,0 & 88,3 & 96,5 & 94,0 \\
\hline PIB per capita * U\$S (2003) & 11.410 & 7.510 & 4.690 & 7.980 & 2.490 & 9.810 & 4.750 \\
\hline \multicolumn{8}{|l|}{ \% población debajo línea de } \\
\hline pobreza internacional (1995-2002) & 3,3 & 8,2 & 16,4 & 2,0 & 14,4 & 2,0 & 14,3 \\
\hline Relación: 20\% más rico/20\% más pobre (1998-2002) & 18,1 & 26,4 & 27,8 & 10,4 & 12,3 & 18,7 & 17,9 \\
\hline $\mathrm{IDH} * \star$ & 0,863 & 0,792 & 0,755 & 0,840 & 0,687 & 0,854 & 0,772 \\
\hline
\end{tabular}

Fuente: Organización Panamericana de la Salud 6.

PBI: Producto Interno Bruto.

* Paridad de poder de compra;

** Índice de Desarrollo Humano 7.

Indicadores de salud seleccionados en los países del MERCOSUR.

\begin{tabular}{|c|c|c|c|c|c|c|c|}
\hline \multirow[t]{2}{*}{ Indicador } & \multicolumn{4}{|c|}{ Estados partícipes } & \multicolumn{2}{|c|}{ Observadores } & \multirow{2}{*}{$\begin{array}{l}\text { En proceso } \\
\text { de integración } \\
\text { Venezuela }\end{array}$} \\
\hline & Argentina & Brasil & Paraguay & Uruguay & Bolivia & Chile & \\
\hline \multirow[t]{2}{*}{ Mortalidad infantil * } & 16,5 & 25,1 & 19,4 & 15,0 & 54,0 & 7,8 & 18,5 \\
\hline & (2003) & $(2002)$ & (2003) & (2003) & $(1999-2003)$ & (2003) & (2003) \\
\hline \multirow[t]{2}{*}{ Mortalidad materna ** } & 43,6 & 73,1 & 174,1 & 23,0 & 230,0 & 13,4 & 57,8 \\
\hline & (2003) & $(2002)$ & $(2003)$ & $(1995-2002) * \star \star$ & (1999-2003) & (2003) & $(2003)$ \\
\hline \multicolumn{8}{|l|}{ Mortalidad ajustada enfermedades } \\
\hline transmisibles (2000-2005) \# & 60,9 & 72,4 & 88,3 & 37,8 & 211,5 & 39,8 & 56,5 \\
\hline \multicolumn{8}{|l|}{ Mortalidad ajustada neoplasmas } \\
\hline malignos (2000-2005) \# & 127,3 & 109,8 & 125,5 & 158,9 & 168,8 & 120,9 & 95,9 \\
\hline \multicolumn{8}{|l|}{ Mortalidad ajustada enfermedades } \\
\hline del aparato circulatorio (2000-2005) \# & 208,5 & 211,0 & 229,2 & 203,8 & 269,2 & 143,9 & 211,0 \\
\hline
\end{tabular}

Fuente: Organización Panamericana de la Salud 6.

* Por 1.000 nacidos vivos;

** Por 100.000 nacidos vivos;

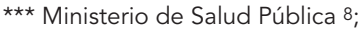

\# Por 100.000

giaba los servicios de alta complejidad (suponiendo la venta internacional de los mismos) o los servicios de promoción y prevención de salud (lo que correspondería a una integración de servicios y portabilidad de derechos), sin que se haya definido claramente cual debe ser su orientación. En cuanto al nivel de las normas de habilitación de servicios de salud, se optó primero por definir "requisitos mínimos", luego por identificar cuales son los "requisitos comunes” entre los cuatro países, pero es evidente que estas soluciones no resultan satisfactorias ni permitirán en un futuro la venta o la integración de servicios, ni su garantía de calidad, 
Recursos destinados a la salud en países seleccionados.

\begin{tabular}{|c|c|c|c|c|c|c|c|}
\hline \multirow[t]{2}{*}{ Indicador } & \multicolumn{4}{|c|}{ Estados partícipes } & \multicolumn{2}{|c|}{ Observadores } & \multirow{2}{*}{$\begin{array}{c}\text { En proceso } \\
\text { de integración } \\
\text { Venezuela }\end{array}$} \\
\hline & Argentina & Brasil & Paraguay & Uruguay & Bolivia & Chile & \\
\hline \% PIB destinado a salud (público) & 5,0 & 3,6 & 2,9 & 2,0 & 4,3 & 2,9 & 1,4 \\
\hline \% PIB destinado a salud (privado) & 3,9 & 3,6 & 4,1 & 10,4 & 2,9 & 3,8 & 2,7 \\
\hline Médicos/10.000 (2001) & 32,1 & 20,6 & 5,6 & 39,0 & 7,6 & 11,5 & 20,0 \\
\hline
\end{tabular}

Fuente: Organización Panamericana de la Salud 6.

PIB: Producto Interno Bruto.

por lo que en la práctica cada país continuaría utilizando sus propias normas.

b) Sub Comisión de Desarrollo y Ejercicio Profesional. Tras un período en el cual esta sub-comisión identificó las especialidades médicas y los integrantes del equipo de salud comunes en los cuatro países, así como los mecanismos de habilitación para el ejercicio profesional, no ha podido avanzar en el tema de reconocimientos mutuos. El grupo de educación del MERCOSUR sí parece haber adelantado en este respecto, pero sin tomar en cuenta el factor habilitación para el ejercicio y el papel de los colegios profesionales. En este momento se encuentra especialmente abocada a la elaboración de una matriz de datos básicos comunes que en el futuro se podrán intercambiar entre los cuatro países. Nuevamente por las características de los países, mientras que Brasil plantea comenzar a nivel de municipios de frontera, los restantes países esperan poder recabar estos datos a nivel nacional. Suponiendo que uno de los objetivos de esta matriz es verificar la habilitación para el ejercicio en una hipótesis de libre tránsito de profesionales de la salud, la limitación a los municipios fronterizos, aunque realista, no parece suficiente.

No se ha planteado el análisis del impacto que una posible liberalización del tránsito de profesionales tendría sobre los sistemas de salud de los Estados partícipes. Dado el desequilibrio existente en la oferta y demanda de profesionales, así como en las condiciones de trabajo y niveles de remuneración entre los países del bloque, podría plantearse un escenario de migración similar al que hoy objeto de atención mundial entre algunos países europeos y sus ex-colonias en África y el Caribe.

c) Sub Comisión de Evaluación y Uso de Tecnologías en Servicios de Salud. Esta sub comisión también ha cambiado sus objetivos en el tiem- po. En la primera pauta negociadora se incluían temas como tecnologías apropiadas, que más tarde fueron abandonados. Se centró luego el trabajo en la armonización de metodologías de evaluación de tecnologías sanitarias, lo que se realizó, pero no se han llevado a cabo evaluaciones conjuntas, a pesar de existir el planteamiento, entre otros motivos porque los sistemas de salud utilizan distintos criterios para la incorporación de prestaciones en sus canastas de servicios y no existe una cobertura uniforme en los cuatro países.

\section{Conclusiones}

De lo anterior se concluye que el proceso de integración en salud se encuentra aún débil y sujeto a una serie de condiciones externas de gran importancia, por lo que es de suponer que será lento. La principal de estas condiciones es el grado de avance que pueda alcanzar la integración política y económica, que al escribir este documento se encuentra cuestionada, especialmente por los países más pequeños del bloque. Las expectativas son altas para un proceso que lleva solamente 15 años, y deberían ajustarse en función de los tiempos requeridos por otros procesos de integración.

Debido a la misma lógica de su creación, se ha avanzado más en todo lo relativo a productos para la salud que en servicios de salud, a pesar de existir acuerdo sobre su importancia.

Entre las carencias más importantes se identificó la de un modelo/imagen objetivo de integración explícito, que pueda orientar el proceso de discusión en los temas de salud y servicios de salud.

Probablemente, debido a la ausencia de esa imagen objetiva, aún no se ha dado la discusión sobre portabilidad de derechos de los ciudada- 
nos, pero es probable que el proceso de integración económica y laboral avance más velozmente que el de salud, por lo que nuestros sistemas de salud podrían llegar a enfrentarse al desafío de responder a esta nueva situación sin directivas claras y consensuadas.

\section{Resumen}

Se describen los antecedentes institucionales de integración en América Latina, tanto económica como en salud y se propone una sistematización de las posibles modalidades de integración en salud. Se identifican factores facilitadores e inhibidores del proceso de integración según cada modalidad y se discute su viabilidad en el presente contexto. Se presenta una breve descripción de la estructura y funcionamiento de las estructuras de salud del MERCOSUR (Reunión de Ministros y Subgrupo de Trabajo 11), así como el grado de avance alcanzado hasta el presente, reflexionando sobre las posibles causas del avance desigual en las diversas áreas.

Sistemas de Salud; Servicios de Salud; Acción Intersectorial

\section{Referencias}

1. Rígoli F. MERCOSUR: marco general e implicaciones para el desarrollo de los recursos humanos. In: Reunión Regional Recursos humanos: factor crítico de la reforma sectorial en salud. San José: Organización Panamericana de la Salud; 1997.

2. Fundación ISALUD. Perspectivas para el Mercado Latinoamericano de Medicamentos. In: Encuentro Subrregional del Cono Sur. Montevideo: Sindicato Médico del Uruguay/Red Interamericana sobre Economía y Financiamiento de la Salud; 1996. p. 12.

3. Comisión de Economía y Salud, Sindicato Médico del Uruguay/Red Interamericana sobre Economía y Financiamiento de la Salud. Procesos de integración en el Mercado Común del Sur. In: Encuentro Subrregional del Cono Sur. Montevideo: Sindicato Médico del Uruguay/Red Interamericana sobre Economía y Financiamiento de la Salud; 1996. p. 230.

4. Hirst M. La dimensión política del MERCOSUR: actores, politización e ideología. Estud Av 1996; 10:217-50.

5. Bertinato L, Busse R, Fahy N, Legido-Quigly H, McKee M, Palm W, et al. Cross border health care in Europe: patient mobility. Brussels: European Observatory on Health Systems and Policies, World Health Organization; 2005. (Policy Brief 9)

6. Organización Panamericana de la Salud. Health situation in the Americas basic indicators 2005. Washington DC: Organización Panamericana de la Salud; 2005

7. Programa de las Naciones Unidas para el Desarrollo. Human development report 2005. http://www. undp.org (accedido el 14/Mar/2006).

8. Ministerio de Salud Pública. Informe del Departamento de Estadística: servicio de información poblacional. Montevideo: Ministerio de Salud; 2003.

Recibido el 14/Jun/2006

Versión final presentada el 14/May/2007

Aprobado el 15/May/2007 